\title{
SCALED AND STABLE MEAN-VARIANCE-EVaR PORTFOLIO SELECTION STRATEGY WITH PROPORTIONAL TRANSACTION COSTS
}

\author{
Ebenezer Fiifi Emire ATTA MILLS', Bo YU², Jie YU ${ }^{3}$ \\ School of Mathematical Sciences, Dalian University of Technology, Dalian, China, 116024 \\ E-mails: 1attamills2003@gmail.com (correspondingauthor); \\ 2yubo@dlut.edu.cn; 3yujie@mail.dlut.edu.cn \\ Received 02 February 2017; accepted 11 June 2017
}

\begin{abstract}
This paper studies a portfolio optimization problem with variance and Entropic Value-at-Risk (EVaR) as risk measures. As the variance measures the deviation around the expected return, the introduction of EVaR in the mean-variance framework helps to control the downside risk of portfolio returns. This study utilized the squared $l_{2}$-norm to alleviate estimation risk problems arising from the mean estimate of random returns. To adequately represent the variance-EVaR risk measure of the resulting portfolio, this study pursues rescaling by the capital accessible after payment of transaction costs. The results of this paper extend the classical Markowitz model to the case of proportional transaction costs and enhance the efficiency of portfolio selection by alleviating estimation risk and controlling the downside risk of portfolio returns. The model seeks to meet the requirements of regulators and fund managers as it represents a balance between short tails and variance. The practical implications of the findings of this study are that the model when applied, will increase the amount of capital for investment, lower transaction cost and minimize risk associated with the deviation around the expected return at the expense of a small additional risk in short tails.
\end{abstract}

Keywords: Value-at-Risk, transaction costs, estimation risk, portfolio optimization, scaled and stabilized portfolio, downside risk.

JEL Classification: G11, G2, O16, D81.

\section{Introduction}

In finance, a portfolio is a collection of assets such as stocks, bonds, and cash equivalents among others. The core problem in wealth allocation is optimal portfolio selection strategy and its concerned with finding a balance between risk and return. The standard portfolio optimization or mean-variance portfolio optimization model proposed by Markowitz (1952) identifies the optimal allocation of assets among a basket of investments and measures optimality based on expected returns and risk (variance) trade-off. The celebrated mean-variance (MV) portfolio optimization model is criticized on the basis of using variance as a risk measure and absence of transaction costs. Variance takes 
into consideration returns exceeding the mean value. It penalizes positive and negative deviations from the mean equally (Markowitz 1968). However, investors are only worried about negative deviations. Borkovec et al. (2010) found out that $40 \%$ of financial market participants credit economic loss in abnormal return to transaction costs. Another shortcoming of MV model is that it's impacted by parameter uncertainty and estimation errors since the mean and the covariance matrix has to be estimated from historical data (Bawa et al. 1979; Merton 1980; Kao, Steuer 2016). The asset returns are regarded as deterministic and represented by a single point estimate, which results in estimation risk. A small perturbation in the input parameters of Markowitz's model leads to a major structural change of the resulting portfolios (Merton 1980; Best, Grauer 1991; Black, Litterman 1992; Ceria, Stubbs 2006; Chopra, Ziemba 2011; Filomena, Lejeune 2012).

The idea of a coherent risk measure was first investigated by Artzner et al. (1999) and has since been used as a tool for establishing the properties of a good risk measure. Empirical research has led to the proposal of alternative risk measures for portfolio optimization. As a coherent risk measure, Conditional Value-at-Risk (CVaR) provides better measures of downside risk than Value-at-Risk, takes into consideration the probability and size of the loss and allows for asymmetric distributions aside having desirable properties of coherence. Rockafellar and Uryasev (2002) studied a broad description of methods for minimizing CVaR and its related optimization problems with CVaR constraints. The mean-CVaR (MC) framework has been investigated in recent research (Agarwal, Naik 2006; Ahmed 2006; Yao et al. 2013; Dai, Wen 2014; Zhao et al. 2015; Moazeni et al. 2016).

Roman et al. (2007) investigated the mean-variance-CVaR (MVC) optimization model and concluded that the MVC model does not dismiss both MV and MC models but embeds them and that the resulting solutions are efficient for mean-variance-CVaR. The mean-variance-CVaR model strikes a balance between obtaining large CVaR from portfolios obtained with mean-variance and large variance from portfolios obtained with mean-CVaR. One can get a better model of the portfolio by employing the three indexes i.e. MVC instead of two parameters (MV or MC) so as to increase the efficiency of the model (Roman et al. 2007). Atta Mills et al. (2016a) investigated a combined risk measure of variance and a modified safety-first principle based on CVAR and VaR and obtained better out-of-sample performance. However, CVaR has a disadvantage of depending only on the tail of the distribution, i.e. it is a $0-1$ risk measure, and as such, it is not smooth (Cherny, Madan 2006). Ahmadi-Javid (2012) showed that stochastic optimization problems that are computationally intractable with CVaR are efficiently deduced when Entropic-Value-at-Risk (EVaR) is considered. Zheng and Chen (2014) studied a portfolio selection strategy based on EVaR and compared the results with other downside risk measures such as VaR and CVaR. Their results show that EVaR has the highest resolution of risk. Various risk measures highlight diverse features of the random loss. In this study, we use variance and EVaR indexes. This controls both the symmetric risk measure and asymmetric downside risk at the tail part of losses. This practically minimizes risk and controls downside risk of portfolio return. An optimal portfolio solution obtained by the MV model may be considered unacceptable by a regulator, since 
it may have a large EVaR, resulting in big losses under undesirable scenarios. Fund managers may also regard a portfolio obtained with a mean-EVaR model unfavourable since it may have a large variance and therefore a small Sharpe index. In this paper, we seek to meet the requirements of the regulator and the fund manager. This provides an answer as to the necessity for considering variance along with EVaR.

To lessen the undesired effect of estimation risk, models based on robust optimization (Goldfarb, Iyengar 2003; Tütüncü, Koenig 2004), factor models (Green, Hollifield 1992; Nagai 2003), stochastic programming (Rockafellar, Uryasev 2000), shrinkage estimators (Jorion 1986; Ledoit, Wolf 2004), have been studied. Another method is the modification of portfolio decision variables by adding regularizers or additional constraints to the optimization problem (Jagannathan, Ma 2003; DeMiguel et al. 2009; Brodie et al. 2009; Atta Mills et al. 2016a; Caccioli et al. 2016). In DeMiguel et al. (2009), an addition of a convex norm ball constraint to the portfolio weight was done. Results revealed that norm ball constrained portfolios have better out of sample performance than the portfolio strategies of the naive $1 / \mathrm{N}$ diversification. The norm ball constraint employed by DeMiguel et al. (2009) acts as a solution to tackle the problems of extreme weights and under-diversification of the portfolio aside estimation risk. The weight constrained portfolio selection strategy is pursued in this study by specifying the general norm as squared $l_{2}$-norm ball.

We study the weight-constrained portfolio selection strategy by shrinking portfolio weights directly. This is prudent for three reasons. First, Fan et al. (2012) demonstrated that the estimation risk is bounded by a function of the norm of portfolio weights, and therefore constraining portfolio norms is equivalent to constraining estimation risks. Second, errors from estimation may arise through mathematical computations in solving for portfolio weights. By working on the portfolio weights directly, their desired forms and characteristics could be achieved. Furthermore, the magnitude of portfolio weights is a proxy for the transaction cost (Brodie et al. 2009).

To rightly represent the consolidated risk measure of the resulting portfolio, this study investigates rescaling by funds available after paying transaction costs (Mitchell, Braun 2013). In this paper, we present a scaled regularized mean-variance-EVaR in which the uncertainty in returns is taken into account by considering stock returns as random variables. In the real world, setting up a new portfolio or revising an existing portfolio requires cost to be incurred and must be inclusive in practical analysis (Chen et al. 1971). Proportional transaction costs (Kellerer et al. 2000; Muthuraman, Kumar 2006; Zhu 2017) which are induced by tax, liquidity costs, brokerage fees (Dumas, Luciano 1991; Kellerer et al. 2000; Lobo et al. 2007; Wang et al. 2017) are incorporated into our scaled regularized mean-variance-EVaR model with an implicit assumption that transaction costs are paid at the beginning of the planning period.

The main novelty of this paper is characterized by the combination of variance and Entropic Value-at-Risk as risk measures in the presence of proportional transaction costs to obtain scaled stable portfolios using squared $l_{2}$-norm regularization technique. This particular perspective and approach have not been explored to the very best of our knowledge. 
This paper is organized as follows: in the next sections, the literature review is discussed, and coherent risk measures are considered. The portfolio selection strategy is presented, and convex reformulation derived. Numerical examples and computational results of our method are shown in the subsequent section. The last section offers concluding remarks.

\section{Literature review}

Markowitz's MV model has been modified and advanced in several directions. Mathematically, Markowitz' MV model produces a quadratic optimization problem:

$$
\begin{array}{ll}
\min & x^{T} Q x \\
\text { subject to } & \mu^{T} x \geq R, \\
& A x=b, \\
& C x \geq d,
\end{array}
$$

where $\mu$ denotes the expected return of assets, $Q$ be the covariance matrix of portfolio return and $R$ is a required return level. The set of admissible portfolios is a nonempty polyhedral set $\Lambda:=\{x: A x=b, C x \geq d\}$. Specifically, one of the constraints in the set $\Lambda$ is $e^{T} x=1$, where $e$ is a vector of ones. Hester and Tobin (1967) introduced the addition of risk-free assets in the basic MV model by the concept of separation theorem, which states that the optimal risk portfolio can be attained without any understanding or knowledge of the investor's preference in the presence of a risk-free financial asset.

An underlying assumption is that the investor has sufficient historical data and that the dynamics of financial assets can be predicted accurately with the historical data. Researchers have detailed the high sensitivity nature of the mean-variance estimates (Bawa et al. 1979). Jobson and Korbie (1980) elaborate these sensitivity problems and propose the application of shrinkage estimators. Random fuzzy models provide some approaches in tackling the uncertainty in returns. León et al. (2002) and Vercher et al. (2007) employ fuzzy numbers as a replacement for uncertain returns of the financial assets. Filomena and Lejeune (2012) made use of probabilistic distributions to model asset returns as random variables in an effort to tackle estimation risk. They presented the probabilistic constraint

$$
\mathbb{P}\left(\sum_{k=1}^{N}\left(\zeta_{k} x_{k}\right) \geq R\right) \geq 1-\epsilon,
$$

where $\zeta_{k}$ is the random return of asset $k, x_{k}$ is amount invested in asset $k$ and $\epsilon$ is the confidence interval. The probabilistic constraint requires the return of the portfolio to exceed the required return level $R$ with probability at least $1-\epsilon$.

The single index model studied by Sharpe (1964) and Lintner (1965) ignores the covariance between the returns of financial assets. The single index model is based on the premise that returns of the financial assets are dependent only on a market index and that the covariance estimate is not required. This further paved the way for the introduction 
of Capital Asset Pricing Model (CAPM). However, CAPM assumes that all investors CAPM's risk measure $\beta$ does not fully measure the risk of most stocks. CAPM states that the expected return of a financial asset or portfolio equals the return on a riskless or risk-free asset plus a risk premium. Mathematically, CAPM is given by:

$$
\mathbb{E}\left[\zeta_{k}\right]=R_{f}+\beta_{k}\left[\mathbb{E}\left[R_{M}\right]-R_{f}\right]
$$

where, $\beta_{k}=\frac{\operatorname{covariance}\left(\zeta_{k}, R_{M}\right)}{\operatorname{variance}\left(R_{M}\right)}, R_{M}$ is return on market portfolio and $R_{f}$ is return on
risk free asset. Several studies have been done on the MV model and associated risk measures. Konno et al. (1993) introduced the mean-absolute deviation (MAD) as an alternative to the use of squared deviations in the MV model. Instead of using squared deviations as in the MV model, Konno et al. (1993)'s dispersion measure is based on the absolute deviations from the mean; that is, the optimization problem is defined as:

$$
\begin{array}{ll}
\max & \mu^{T} x \\
\text { subject to } & \mathbb{E}\left(\left|\sum_{k=1}^{N} x_{k} \zeta_{k}-\sum_{k=1}^{N} x_{k} \mu_{k}\right|\right) \geq b, \\
& e^{T} x=1 .
\end{array}
$$

Advancements have led to minimizing semivariance (Huang 2008; Markowitz et al. 1993). The portfolio semivariance is defined as:

$$
\mathbb{E}\left(\min \left(\sum_{k=1}^{N} x_{k} \zeta_{k}-\sum_{k=1}^{N} x_{k} \mu_{k}, 0\right)\right)^{2}
$$

where $\zeta_{k}$ is the random return for asset $k=1, \ldots, N$. The concept of skewness in the portfolio selection strategy has also been considered (Konno, Suzuki 1995; Chunhachinda et al. 1997). Other risk measures considered are entropy (Philippatos, Wilson 1972), lower partial moments (Price et al. 1982), Gini mean difference (Shalit, Yitzhaki 1984), Value-at-Risk (Gaivoronski, Pflug 2005) and Conditional Value-at-Risk (CVaR) (Rockafellar, Uryasev 2002) among others. Several studies have proposed the fusion of two or more risk measures and obtained better portfolios (Roman et al. 2007; Atta Mills et al. 2016a) and provided answers to the inconsistencies in the mean-variance and mean-CVaR models. Mathematically, Roman et al. (2007)'s approach is presented as:

$$
\begin{array}{ll}
\min & x^{T} Q x \\
\text { subject to } & \mathrm{CVaR}_{(1-\epsilon)}(x) \leq b, \\
& \mu^{T} x \geq R, \\
& e^{T} x=1,
\end{array}
$$

where $1-\epsilon$ denotes confidence level, $b$ and $R$ are real numbers denoting required level of risk and required level of returns respectively. CVaR has a disadvantage of depending only on the tail of the distribution as such; it is not smooth (Cherny, Madan 2006). 
Ahmadi-Javid (2012) showed that stochastic optimization problems that are computationally intractable with CVaR are efficiently deduced when Entropic-Value-at-Risk (EVaR) is considered. Zheng and Chen (2014) studied a portfolio selection strategy based on EVaR and compared the results with other downside risk measures such as VaR and CVaR. Their results show that EVaR has the highest resolution of risk. In this paper, we seek to meet the requirements of the regulator (small short tails) and the fund manager (small variance). This provides an answer as to the necessity for considering variance along with EVaR. Employing variance and EVaR helps control symmetric and assymetric risk on portfolio returns.

To solve the MV model and its modifications, several methods have been employed which includes but not limited to linear goal programming (Sharpe 1967), Genetic Algorithms (Loraschi et al. 1995), Fuzzy modeling (Tanaka, et al. 2000), particle swarm optimization (Kendall, Su 2005), stochastic programming (Samuelson 1969; Atta Mills et al. 2016b) and evolutionary algorithm (Chiam et al. 2009).

For portfolio management in practice, the standard mean-variance only serves as a starting point. It is reasonable to amend the underlying portfolio selection strategy framework with different types of restrictions that take institutional features and investment guidelines into consideration. In practical portfolio management, a portfolio manager is faced with some constraints as a result of legal restraints, industrial regulations, and client-initiated strategies among others. A portfolio manager may be faced with a restriction of the minimum capital allocation to a particular asset. As a result, the underlying portfolio selection strategy must reflect some of these real-world constraints such as such as transaction costs, minimum transaction lots, cardinality constraints, and thresholds on maximum or minimum investments. The importance of real features in a portfolio selection model has been investigated in Kellerer et al. (2000). It is shown that the use of fixed transaction costs lowers the number of securities selected and that employing transaction lots considerably alters the structure of the resulting portfolio, both regarding assets selected and capital invested in the assets. One of the first studies on the inclusion of transaction costs in the mean-variance framework was in 1970 by Pogue (1970). Further extensions and modifications have been investigated afterwards (Schreiner 1980; Lobo et al. 2007; Mitchell, Braun 2013).

When securities are bought and sold, expenses are incurred such as bid-ask spreads and fund loads or brokerage fees. The sum of transaction costs for each trade is given by:

$$
c(x)=\sum_{i=1}^{N} c_{i}\left(x_{i}\right),
$$

where $c_{i}$ is the transaction cost function for asset $i$. A simple situation for transaction cost model is to make the assumption that there exist a perfect market i.e. $c(x)=0$. Another variant of practical importance is that transaction costs are linear, with the costs of each transaction proportional (Kellerer et al. 2000; Muthuraman and Kumar 2006) to the tradable amount:

$$
c_{i}\left(x_{i}\right)= \begin{cases}c_{i}^{b} x_{i}, & x_{i} \geq 0 \\ c_{i}^{s} x_{i}, & x_{i} \leq 0 .\end{cases}
$$


Here, $c_{i}^{b}$ and $c_{i}^{s}$ are the costs incurred when buying and selling asset $i$ respectively. Linear costs can be handled with the introduction of new variables $x^{b} \geq 0$ and $x^{s} \geq 0$, which represents the portion of capital used to purchase asset $i$ and portion of capital obtained by selling shares of asset $i$. Thus, the total transaction can be expressed as:

$x_{i}=x_{i}^{b}-x_{i}^{s}$.

Therefore, the transaction cost function $c_{i}$ is given as:

$c_{i}=c_{i}^{b} x_{i}^{b}+c_{i}^{s} x_{i}^{s}$.

A fixed cost per transaction might be imposed as studied by Oksendal and Sulem (2002). Transaction costs can be modeled as fixed costs plus proportional costs (Maringer 2006). Let $\beta_{i}^{b}$ and $\beta_{i}^{s}$ be the fixed costs per transaction for buying and selling asset $i$ respectively. The fixed-plus-proportional costs function is expressed as:

$$
c_{i}\left(x_{i}\right)= \begin{cases}0, & 0 \\ \beta_{i}^{b}+c_{i}^{b} x_{i}, & x_{i}>0 \\ \beta_{i}^{s}+c_{i}^{s} x_{i}, & x_{i}<0 .\end{cases}
$$

The function above is non-convex except for when the fixed costs are zero. Alternatively, transaction costs could be modeled as proportional cost with a lower limit being the fixed cost. This can be represented by a cost function:

$$
c_{i}\left(x_{i}\right)= \begin{cases}0, & 0 \\ \max \left\{\beta_{i}^{b}, c_{i}^{b} x_{i}\right\}, & x_{i}>0 \\ \max \left\{\beta_{i}^{s}, c_{i}^{s} x_{i}\right\}, & x_{i}<0 .\end{cases}
$$

As already stated in the previous section, this study assumes proportional transaction costs. The transaction cost models have no advantages over each other as the assumption and selection of which transaction cost model to employ depends on the researcher. For a detailed review of risk measures, the basic solvable portfolio models and models with real features that are presented in the literature, please refer to Mansini et al. (2014) and Ghosh and Mahanti (2014). All these later developments can be categorized into three sections: (i) developing models to reflect investor's preferences, (ii) inclusion of real-world market constraints and (iii) using characteristics of diverse disciplines to solve practical portfolio selection strategy problems.

\section{Coherent risk measure}

Consider $F^{\infty}$ as a set of all bounded random variables denoting financial positions.

Definition 1. (Follmer, Schied 2004) For a risk measure $\rho: F^{\infty} \rightarrow \mathbb{R}$ to be referred to as a coherent risk measure, the following properties must be satisfied given that $X$ and $Y$ are random variables:

(1) Monotonicity. If $X \geq 0$, then for any $X \in F^{\infty}$.

(2) Positive homogeneity. $\rho(s X)=s \rho(X)$ for any $X \in F^{\infty}$ and $s>0$.

(3) Sub-additivity. $\rho(X+Y) \leq \rho(X)+\rho(Y)$ for any $X, Y \in F^{\infty}$. 
(4) Translational invariance. $\rho(X+s) \leq \rho(X)-s$ for any $X \in F^{\infty}$ and $s \in \mathbb{R}$.

From here on, we discuss CVaR and EVaR. For a comprehensive study on CVaR and EVaR, please see Rockafellar and Uryasev (2000) and Ahmadi-Javid (2012) respectively.

Definition 2. Define loss of the portfolio function as $f(X, \zeta)$ with decision vector $x \in X \subseteq \mathbb{R}^{N}$ and random vector which is the actual return of the portfolio. Suppose $\mathbb{E}(|f(x, \zeta)|)$ for each $x \in \mathbb{R}^{N}$ and $\zeta$ has a c.d.f $p(\zeta)$, then under a confidence level $\epsilon(0<\epsilon<1)$ and a threshold $z$, we define Value-at-Risk (VaR) and CVaR as follows:

$$
\begin{aligned}
& \operatorname{VaR}_{(1-\epsilon)}(x)=\min \left\{z \mid \int_{f(x, \zeta) \leq z} p(\zeta) d \zeta \geq 1-\epsilon\right\} . \\
& \operatorname{CVaR}_{(1-\epsilon)}(x)=\frac{1}{1-(1-\epsilon)} \int_{f(x, \zeta) \geq \operatorname{VaR}_{(1-\epsilon)}(x)} f(x, \zeta) p(\zeta) d \zeta .
\end{aligned}
$$

Equivalently, Rockafellar and Uryasev (2000) proved that $\operatorname{CVaR}_{(1-\epsilon)}(x)$ can be defined as $\operatorname{CVaR}_{(1-\epsilon)}(x)=\min _{z \in \mathbb{R}} F_{(1-\epsilon)}(x, z)$,

where $F_{(1-\epsilon)}(x, z)=z+\frac{1}{1-(1-\epsilon)} \int_{\zeta \in \mathbb{R}^{N}}[f(x, \zeta)-z]^{+} p(\zeta) d \zeta$ or for the discrete case $F_{(1-\epsilon)}(x, z) \approx z+\frac{1}{N(1-(1-\epsilon))} \sum_{k=1}^{N}\left[f\left(x, \zeta_{k}\right)-z\right]^{+}$.

Therefore, minimizing $\operatorname{VaR}_{(1-\epsilon)}(x)$ w.r.t $x \in X$ is equivalent to minimizing $F_{(1-\epsilon)}(x, z)$ w.r.t $(x, z) \in X \times \mathbb{R}$ i.e.,

$$
\min _{z \in \mathbb{R}} \operatorname{CVaR}_{(1-\epsilon)}(x)=\min _{(x, y) \in X \times \mathbb{R}} F_{(1-\epsilon)}(x, z) .
$$

CVaR has a disadvantage as it depends only on the tail of the distribution, i.e. it is a $0-1$ risk measure so it is not smooth (Cherny, Madan 2006). Ahmadi-Javid (2012) showed that stochastic optimization problems that are computationally intractable with CVaR are efficiently deduced when Entropic-Value-at-Risk (EVaR) is considered. According to Axelrod et al. (2016), EVaR is a computationally efficient and coherent risk measure that can be utilized to quantify risk.

For the purpose of this study, we investigate the Entropic-Value-at-Risk (EVaR) as part of a consolidated risk measure with variance in a mean-risk optimization framework to help control the downside risk of the portfolio return.

Definition 3. (Ahmadi-Javid 2012) For a given level of confidence $\epsilon \in(0,1)$, the Entropic Value-at-Risk of a random variable $X$ whose distribution $\mathbb{P}$ belongs to set $\mathbb{Q}$ of distributional ambiguity is defined as

$$
\operatorname{EVaR}_{(1-\epsilon)}(X)=\inf _{z>0}\left\{\frac{1}{z}\left[\ln \mathbb{E}_{p}\left[e^{z X}\right]-\ln \epsilon\right]\right\} .
$$

EVaR's dual representation relates to the concept of entropy. Ahmadi-Javid (2012) investigates the EVaR with a known distribution and from a risk measure viewpoint. They prove EVaR is a coherent risk measure and thus has all the desirable properties defined 
above. EVaR is a coherent risk measure that corresponds to the tightest possible upper bound obtained from the Chernoff inequality for both the Value-at-risk (VaR) and the Conditional Value-at-Risk (CVaR).

We assume that $X$ follows a Gaussian distribution with mean $\mu$ and standard deviation $\sigma$. This leads to a recast of (11):

$$
\begin{aligned}
& \operatorname{EVaR}_{(1-\epsilon)}(X)=\inf _{z>0}\left\{\frac{1}{z}\left[\ln \mathbb{E}_{X \sim N\left(\mu, \sigma^{2}\right)}\left[e^{z X}\right]-\ln \epsilon\right]\right\}= \\
& \inf _{z>0}\left\{\mu+\frac{\sigma^{2}}{2 z}-\frac{1}{z} \ln \epsilon\right\}= \\
& \mu+\sqrt{2 \ln \frac{1}{\epsilon} \sigma .}
\end{aligned}
$$

\section{Constraining portfolio norms}

In the next section, this study investigates portfolios resulting from a scaled mean variance-EVaR strategy but subject to the additional constraint that the norm of the portfolio weight vector is smaller than a given threshold, $\psi$. One can view these portfolios as resulting from shrinking portfolio weights of the scaled mean-variance EVaR portfolio. As already stated, shrinking portfolio weights is prudent and by constraining portfolio norms, we constrain estimation risks. The general $p$-norm, $p \geq 1$ is defined as

$$
\|x\|_{p}=\left(\sum_{i=1}^{N}\left|x_{i}\right|^{p}\right)^{1 / p} \text {. }
$$

When $p=1$, we obtain the taxicab norm $\left(l_{1}\right.$-norm $)$. For $p=2$, we get the Euclidean norm $\left(l_{2}\right.$-norm). Note that the standard mean-variance or mean-EVaR is the solution the norm-constrained problem with $\psi=\infty$. The norm-constrained scaled mean-varianceEVaR is a shrinkage estimator of the scaled mean-variance-EVaR. The difficulty in shrinkage estimators is the selection of the amount of shrinkage that generates optimal portfolios that reflects the trade-off between returns and risk. DeMiguel et al. (2009) provided a general scheme for obtaining optimal portfolios by adding a convex norm ball constraint. The numerical results indicated a better out-of- sample performance than strategies like $1 / N$ portfolio strategy among others. Atta Mills et al. (2016a) also employed the Squared-Euclidean norm ball constraint to alleviate estimation risk and obtained better computational results than other strategies.

\section{The model}

We formulate the scaled regularized MVE portfolio revision model with transaction costs as a mathematical optimization problem in this section. This study employs the following notation. We denote by $R$ the required return level, $N$ the number of risky assets and $x^{0}$ as the initial risky assets before revision: $x_{k}^{0}$ is the portion of capital initially allocated to asset $k, k=1,2,3, \ldots, N$. Let $x_{k}, x^{b}$ and $x^{s}$ be $N$-dimensional vectors 
of controllable variables: $x_{k}$ is the portfolios invested in risky asset $k$ after revision, $x_{k}^{b}$ are purchases (portion used) of risky asset $k$ and $x_{k}^{s}$ are sales (portion obtained) of risky asset $k$. The transaction costs incurred when selling risky assets is $c^{s}$ and that of buying risky assets is $c^{b}$. The financial portfolio is characterized by $N$-dimensional vector of random returns $\zeta$. Let $\mu_{k}$ denote the expected value of $\$ 1$ invested in risky asset $k$ at the end of the period and $\mu_{f}>1$ be the value of riskless asset at the end of the period, $y^{0}$ is the fraction invested in a riskless asset before revision and $y$ is the fraction invested in a riskless asset after revision. The variance is denoted by $\sigma^{2}(\zeta)=E\left[(\zeta-E(\zeta))^{2}\right]$. Denote $(1-\epsilon)$ the probability risk level. This research uses historical data of returns $r_{k}$ for each risky asset $k$ at $T$ successive time frames to estimate the mean return $\mu$ the covariance matrix, $\left.Q \succeq 0: Q=E[(\zeta-\mu)(\zeta-\mu)]^{T}\right]$. For convenience, the notations are listed below: $N$ - number of risky assets.

$R$ - required return level.

$e-$ vector with entries equal to ones.

$x^{0}-$ initial risky assets before revision.

$x_{k}^{0}$ - portion of capital initially allocated to risky asset $k$.

$x_{k}$ - amount invested in risky asset $k$ after revision.

$x_{k}^{b}$ - purchases (portion used) of risky asset $k$.

$x_{k}^{s}$ - sales (portion obtained) of risky asset $k$.

$c^{s}$ - transaction costs incurred when selling a risky asset.

$c^{b}$ - transaction costs associated with purchasing a risky asset.

$y$ - amount invested in the riskless asset before revision.

$y$ - amount invested in the riskless asset after revision.

$\mu_{k}$ - expected return of $\$ 1$ invested in risky asset $k$.

$\mu_{f}$ - expected return of riskless asset.

$Q$ - covariance matrix of the portfolio return.

\subsection{Regularized mean-variance-EVaR model}

To this end, we consider the squared $l_{2}$-norm regularized mean-variance-EVaR portfolio optimization model with proportional transaction costs as the optimization problem $P_{0}$.

$$
\begin{array}{ll}
P_{0}: \min & x^{T} Q x+\operatorname{EVaR}_{(1-\epsilon)} x \\
\text { subject to } & \mu_{f} y+\mu^{T} x \geq R, \\
& y+\sum_{k=1}^{N} x_{k}+c^{b} \sum_{k=1}^{N} x_{k}^{b}+c^{s} \sum_{k=1}^{N} x_{k}^{s} \leq 1, \\
& x_{k}=x_{k}^{0}+x_{k}^{b}-x_{k}^{s}, \quad \forall k, \\
& x_{k}^{b} \cdot x_{k}^{s}=0, \\
& \|x\|_{2}^{2} \leq \psi^{2}, \\
& x, y, x^{b}, x^{s} \in \mathbb{R}_{+}^{N},
\end{array}
$$


where $l_{2}$-norm of $x \in \mathbb{R}^{N}$ is defined as $\|x\|_{2}=\sqrt[2]{\sum_{k=1}^{N}\left|x_{k}\right|^{2}}$, the squared $l_{2}$-norm is $\|x\|_{2}^{2}=\sum_{k=1}^{N} x_{k}^{2}$ and $\psi$ is a given threshold.

Problem $P_{0}$ minimizes the squared $l_{2}$-norm-constrained variance-EVaR of the portfolio and is subjected to a set of constraints (15-20). Constraint (15) guarantees the expected portfolio return to be greater or equal to a minimum threshold return level $R$. Constraint (16) represents the budget constraint. Constraint (17) is the balance constraints and represents the portfolio position to be selected explicitly through purchase $x^{b}$ and sales $x^{s}$ that are adjustments to the initial position $x^{0}$. The complementarity constraint (18) and the nonnegative constraint (20) prevent the possibility of simultaneous sales and purchases. Practically, concurrently selling and buying is seen as not optimal because making the allocation to one asset or security decreases and increases at the same time, thus being subjected to unnecessary transaction costs (Dybvig 2005). Constraint (19) helps to alleviate the estimation risk problem in the mean estimate (DeMiguel et al. 2009).

In the MVE approach, given two portfolios say $a$ and $b$, portfolio $a$ is favoured over portfolio $b$ if and only if the following conditions hold: $E\left(\zeta_{a}\right) \geq E\left(\zeta_{b}\right), \sigma^{2}\left(\zeta_{a}\right) \leq \sigma^{2}\left(\zeta_{b}\right)$ and $\operatorname{EVaR}\left(\zeta_{a}\right) \leq \operatorname{EVaR}\left(\zeta_{b}\right)$ in which $\operatorname{EVaR}$ is computed accordingly.

Another alternative is to explicitly model the trade-off between return and risk in the objective function using a risk-aversion parameter, $\alpha$. We refer to the following formulation as the risk aversion formulation of optimization problem $P_{0}$ :

$$
\begin{array}{cc}
A_{0}: \max & \mu_{f} y+\mu^{T} x-\alpha_{1} x^{T} Q x-\alpha_{2} \operatorname{EVaR}_{(1-\epsilon)} x \\
\text { subject to } & y+\sum_{k=1}^{N} x_{k}+c^{b} \sum_{k=1}^{N} x_{k}^{b}+c^{s} \sum_{k=1}^{N} x_{k}^{s} \leq 1 \\
& x_{k}=x_{k}^{0}+x_{k}^{b}-x_{k}^{s}, \quad \forall k \\
& x_{k}^{b} \cdot x_{k}^{s}=0 \\
& \|x\|_{2}^{2} \leq \psi^{2}, \\
& x, y, x^{b}, x^{s} \in \mathbb{R}_{+}^{N} .
\end{array}
$$

The risk aversion parameter $\alpha$ is also known as the Arrow-Pratt risk aversion index. When $\alpha$ is small (i.e., the aversion to risk is low), the penalty from the contribution of the portfolio risk is also small, resulting in risky portfolios. Contrarily, when $\alpha$ is large, portfolios with more exposures to risk become more highly penalized. If $\alpha$ is gradually increased from zero and for each case compute the optimization problem, it results in finding each portfolio along the efficient frontier. Often, $\alpha$ is calibrated via backtests with historical data such that a particular portfolio has the desired risk profile.

These formulations $P_{0}$ and $A_{0}$ are equivalent as they lead to the same efficient frontier and trade expected portfolio risk versus portfolio return in a similar manner. For the purpose of this study, we use the risk minimization formulation $P_{0}$ as it provides an avenue to consider varied required returns needed for our numerical examples. 


\subsection{Scaled regularized mean-variance-EVaR approach}

In a mean-variance framework, Mitchell and Braun (2013) proposed rescaling by capital available after incurring transaction costs to obtain an appropriate representation of a risk measure of the resulting portfolio. They obtained a frugal optimal solution to the MV problem. Based on Mitchell and Braun (2013) approach, we scale the regularized variance-EVaR risk measurement by the square of capital amount actually invested. Earlier, we optimized the regularized variance-EVaR i.e.

$$
x^{T} Q x+\operatorname{EVaR}_{(1-\epsilon)} x .
$$

When transaction costs are non-existent in the market, the entire capital (\$1) is always accessible for investment, but in the presence of transaction costs, costs would have to be paid to revise the portfolio, and so the $\$ 1$ is no more available for investment. The suitable objective is therefore represented as

$$
\frac{x^{T} Q x+\operatorname{EVaR}_{(1-\epsilon)} x}{\left(e^{T} x+y\right)^{2}} .
$$

The transaction costs paid amounts to $1-\left(e^{T} x+y\right)$, so $e^{T} x+y$ is the actual capital available for investment. We choose to scale the regularized variance-EVaR measurement by the square of the capital actually invested. When the transaction cost is zero, then $e^{T} x+y=1$ and we regain the regularized variance-EVaR measurement. To this end, we consider the scaled squared $l_{2}$-norm regularized mean-variance-EVaR model

$$
\begin{array}{ll}
S_{0}: \min & \frac{x^{T} Q x+\operatorname{EVaR}_{(1-\epsilon)} x}{\left(e^{T} x+y\right)^{2}} \\
\text { subject to } & \mu_{f} y+\mu^{T} x \geq R, \\
& y+\sum_{k=1}^{N} x_{k}+c^{b} \sum_{k=1}^{N} x_{k}^{b}+c^{s} \sum_{k=1}^{N} x_{k}^{s} \leq 1, \\
& x_{k}=x_{k}^{0}+x_{k}^{b}-x_{k}^{s}, \quad \forall k, \\
& x_{k}^{b} \cdot x_{k}^{s}=0, \\
& \|x\|_{2}^{2} \leq \psi^{2}, \\
& x, y, x^{b}, x^{s} \in \mathbb{R}_{+}^{N} .
\end{array}
$$

The above problem $S_{0}$ is a fractional optimization problem and we will find efficient frontiers as shown in the next section. Our fractional model improves the consolidated risk measure for transaction cost consumed portfolios and leads to an optimal portfolio with lower transaction costs.

Theorem 1. Let $x^{*}$ and $y^{*}$ be the optimal solution to $S_{0}$. Denote $C=1-\left(e^{T} x^{*}+y^{*}\right)$ be transaction costs of this portfolio. The portfolio $x^{*}$ and $y^{*}$ is the minimum risk portfolio in comparison to all other portfolios with expected return at $R$ and transaction costs no greater than $C$. 
Proof. Let $\hat{x}$ and $\hat{y}$ solve $S_{0}$, then the feasible region to $S_{0}$

$$
\frac{x^{* T} Q x^{*}+\operatorname{EVaR}_{(1-\epsilon)} x^{*}}{\left(e^{T} x^{*}+y^{*}\right)^{2}} \leq \frac{\hat{x}^{T} Q \hat{x}+\operatorname{EVaR}_{(1-\epsilon)} \hat{x}}{\left(e^{T} \hat{x}+\hat{y}\right)^{2}} .
$$

It follows that if $e^{T} \hat{x}+\hat{y} \geq e^{T} x^{*}+y^{*}$ then $\hat{x}^{T} Q \hat{x}+\operatorname{EVaR}_{(1-\epsilon)} \hat{x} \geq x^{* T} Q x^{*}+\operatorname{EVaR}_{(1-\epsilon)} x^{*}$. Our model $S_{0}$ produces Pareto optimal solutions with varied return level $R$ and threedimensional efficient frontier. The remainder of the paper is in two folds. First, we obtain a convex formulation equivalent to our proposed model, $S_{0}$. Secondly, we present our results via numerical experiment and make concluding remarks.

\subsection{Convex reformulation}

Model $S_{0}$ has complementarity constraint (18), which leads to non-convex optimization problem. Some studies do not impose the complementarity constraint (Krokhmal et al. 2002; Lobo et al. 2007) on the buy and sell variables which results in an unreasonable portfolio selection strategy. Mitchell and Braun (2004) show that the intractable complementarity constraint (18) can be discarded when the portfolio considers a riskless asset. Mitchell and Braun (2004) show that in a case where the riskless asset appears as a nonnegativity constraint, the optimal solution to $P_{0}$ satisfies the transaction cost constraint (5) at equality. Thus, in this case it is not a necessity to consider the complementarity constraint. In other words, the optimization problem $S_{0}$ with explicit sell and buy variables can be solved through a convex relaxation. Given any feasible solution with $y+\sum_{k=1}^{N} x_{k}+c^{b} \sum_{k=1}^{N} x_{k}^{b}+c^{s} \sum_{k=1}^{N} x_{k}^{s} \leq 1$, an equally good feasible solution with $y+\sum_{k=1}^{N} x_{k}+c^{b} \sum_{k=1}^{N} x_{k}^{b}+c^{s} \sum_{k=1}^{N} x_{k}^{s}=1$ can be achieved through the increment of $y$. Thus,

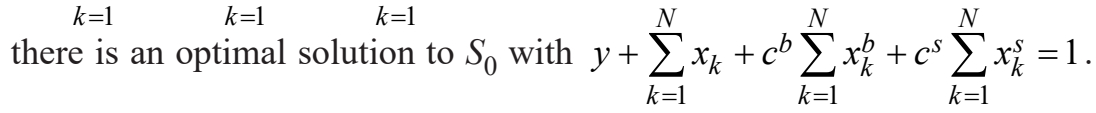

The fractional problem $S_{0}$ can be recast into convex optimization problem by substituting the denominator by the square of the reciprocal of a variable. We provide a convex equivalent reformulation that is mathematically tractable and suitable for solvers. This study employs the technique proposed by Charnes and Cooper (1962) in relation to the solving fractional optimization problems. In Schaible (1974)'s study, an extension of Charnes and Cooper (1962)'s approach was investigated with fractional convex functions incorporated, a scenario which $S_{0}$ is inclusive. Denote

Note that: $\sum_{k=1}^{N} x_{k}+y$ is $e^{T} x+y$.

$$
w:=\frac{1}{e^{T} x+y}, \quad w \geq 1 .
$$

Let's define

$$
\begin{aligned}
& e^{T} x w+y w=1, \\
& \tilde{x}=x w, \quad \tilde{y}=y w, \\
& \tilde{x}^{b}=x^{b} w, \quad \tilde{x}^{s}=x^{s} w .
\end{aligned}
$$


With the complementarity constraint discarded, all the constraints of $S_{0}$ are multiplied by $w$ and with constraint (20) added to the reformulated optimization problem. We also need to impose the constraint $\tilde{y}+\sum_{k=1}^{N} \tilde{x}_{k}=1$, which is equivalent to $w$ (see 23). Thus, we obtain a new convex optimization problem in the form:

$$
\begin{array}{ll}
S_{1}: \min \quad & \tilde{x}^{T} Q \tilde{x}+\operatorname{EVaR}_{(1-\epsilon)} \tilde{x} \\
\text { subject to } & \mu_{f} \tilde{y}+\mu^{T} \tilde{x} \geq R w, \\
& \tilde{y}+\sum_{k=1}^{N} \tilde{x}_{k}+c^{b} \sum_{k=1}^{N} \tilde{x}_{k}^{b}+c^{s} \sum_{k=1}^{N} \tilde{x}_{k}^{s} \leq w, \\
& \tilde{x}_{k}=x_{k}^{0} w+\tilde{x}_{k}^{b}-\tilde{x}_{k}^{s}, \quad \forall k, \\
& \tilde{y}+\sum_{k=1}^{N} \tilde{x}_{k}=1, \\
& \|\tilde{x}\|_{2}^{2} \leq \psi^{2} w, \\
& \tilde{x}, \tilde{y}, \tilde{x}^{b}, \tilde{x}^{s} \in \mathbb{R}_{+}^{N} .
\end{array}
$$

From the above problem, we solve for solution $\tilde{x}^{*}, \tilde{y}^{*}, \tilde{x}^{b^{*}}, \tilde{x}^{s^{*}}$ and $w^{*}$ and obtain an optimal solution to the original problem $S_{0}$ with the complementarity constraint discarded by rescaling $\tilde{x}^{*}, \tilde{y}^{*}, \tilde{x}^{* b}, \tilde{x}^{*} s$ so $x^{*}=\frac{\tilde{x}^{*}}{w^{*}}, y^{*}=\frac{\tilde{y}^{*}}{w^{*}}, x^{b^{*}}=\frac{\tilde{x}^{b^{*}}}{w^{*}}$ and $x^{s^{*}}=\frac{\tilde{x}^{s^{*}}}{w^{*}}$.

\section{Numerical example}

This section illustrates the applicability of the portfolio strategy presented in the previous sections. All computations were performed with MATLAB (version 2015a) software on a Macbook Pro with 2.3 GHz Intel Core i7 processor and 16GB of RAM. The monthly stock price of the top 20 S\&P 500 companies by weight on February 22, 2016 that traded from January 2, 2004 to February 1, 2016 were extracted from Economic Research - St. Louis Fed (2016). For the riskless asset, we consider the 4-Week US Treasury Bill rate on a monthly basis for the same period.

The initial position of stocks $x_{k}^{0}, k=1, \ldots, 20$ is computed by using natural logarithm of the price ratio for 2 consecutive months from January 2, 2004 to December 1, 2004. Assuming the initial position for the 4-Week US Treasury Bill is zero i.e. $y^{0}=0$, the initial position of stocks $x_{k}^{0}, k=1, \ldots, 20$ is obtained by solving the standard meanvariance problem:

$$
\begin{array}{ll}
B_{0}: \min & x^{T} Q x \\
\text { subject to } & \mu_{f} \bar{y}+\mu^{T} \bar{x} \geq R, \\
& \bar{y}+\sum_{k=1}^{N} \bar{x}_{k}=1, \\
& \bar{x}, \in \mathbb{R}_{+}^{N},
\end{array}
$$

where $R=\min (\mu)$ and by setting $x^{0}=\bar{x}^{*}, y^{0}=\bar{y}^{*}$ with $\bar{x}^{*}$ and $\bar{y}^{*}$ denoting the optimal solution of the above model. Please refer to Table 1 for the results. 
Table 1. Initial position of stocks

\begin{tabular}{cc}
\hline Stock $k$ & Initial weight $x^{0}$ \\
\hline 1 & 0.020638 \\
\hline 2 & 0.000004 \\
\hline 3 & 0.241291 \\
\hline 4 & 0.000004 \\
\hline 5 & 0.059342 \\
\hline 6 & 0.000012 \\
\hline 7 & 0.244491 \\
\hline 8 & 0.000004 \\
\hline 9 & 0.128595 \\
\hline 10 & 0.011539 \\
\hline
\end{tabular}

\begin{tabular}{cc}
\hline Stock $k$ & Initial weight $x^{0}$ \\
\hline 11 & 0.000003 \\
\hline 12 & 0.000001 \\
\hline 13 & 0.112752 \\
\hline 14 & 0.000721 \\
\hline 15 & 0.000009 \\
\hline 16 & 0.042769 \\
\hline 17 & 0.130161 \\
\hline 18 & 0.000004 \\
\hline 19 & 0.007657 \\
\hline 20 & 0.000003
\end{tabular}

We construct the portfolio invested in the top 20 S\&P 500 stocks after revision based on natural logarithm of the price ratio for 2 consecutive days from January 3, 2005 to February 1, 2016 given that the amount invested in 4-Week US Treasury Bill after revision is fixed. Descriptive statistics of this data are provided in Table 2.

Considering varied required returns, say, $R=(0.0075,0.0078,0.0082,0.0088,0.0098$, $0.0104,0.0125,0.0130$ ), for $\epsilon=0.05, \psi=0.03$ and transaction costs, $c^{b}=c^{s}=0.02$, the optimal weights of the 20 stocks are obtained by solving model $S_{1}$.

Table 2. Descriptive statistics of 20 S\&P stocks log return distributions

\begin{tabular}{cccccccc}
\hline Stock $k$ & Mean & Std. Deviation & Variance & Skewness & & Kurtosis & \\
\hline & Statistic & Statistic & Statistic & Statistic & Std. error & Statistic & Std. error \\
\hline 1 & 0.0232 & 0.09840 & 0.010 & -1.070 & 0.209 & 3.227 & 0.416 \\
\hline 2 & 0.0068 & 0.06916 & 0.005 & -0.039 & 0.209 & 0.685 & 0.416 \\
\hline 3 & 0.0056 & 0.05147 & 0.003 & 0.230 & 0.209 & 1.204 & 0.416 \\
\hline 4 & 0.0062 & 0.03886 & 0.002 & -0.407 & 0.209 & 1.257 & 0.416 \\
\hline 5 & 0.0012 & 0.08117 & 0.007 & -0.787 & 0.209 & 3.057 & 0.416 \\
\hline 6 & 0.0060 & 0.04863 & 0.002 & -0.038 & 0.209 & 1.276 & 0.416 \\
\hline 7 & 0.0070 & 0.04893 & 0.002 & -0.734 & 0.209 & 0.908 & 0.416 \\
\hline 8 & 0.0053 & 0.04331 & 0.002 & -0.264 & 0.209 & 0.398 & 0.416 \\
\hline 9 & 0.0055 & 0.08960 & 0.008 & -1.164 & 0.209 & 9.445 & 0.416 \\
\hline 10 & 0.0051 & 0.08408 & 0.007 & -0.544 & 0.209 & 1.299 & 0.416 \\
\hline 11 & 0.0066 & 0.05070 & 0.003 & -0.100 & 0.209 & -0.077 & 0.416 \\
\hline 12 & 0.0186 & 0.11104 & 0.012 & -0.015 & 0.209 & 1.904 & 0.416 \\
\hline 13 & 0.0041 & 0.05648 & 0.003 & -0.419 & 0.209 & 0.524 & 0.416 \\
\hline 14 & 0.0079 & 0.04403 & 0.002 & -0.540 & 0.209 & 1.997 & 0.416 \\
\hline 15 & 0.0066 & 0.05901 & 0.003 & -0.322 & 0.209 & 0.074 & 0.416 \\
\hline 16 & 0.0098 & 0.06352 & 0.004 & -0.255 & 0.209 & 0.305 & 0.416 \\
\hline 17 & 0.0070 & 0.04072 & 0.002 & -1.607 & 0.209 & 6.912 & 0.416 \\
\hline 18 & 0.0104 & 0.06391 & 0.004 & -0.553 & 0.209 & 1.166 & 0.416 \\
\hline 19 & 0.0066 & 0.06463 & 0.004 & -0.459 & 0.209 & 1.253 & 0.416 \\
\hline 20 & 0.0082 & 0.07052 & 0.005 & -0.452 & 0.209 & 0.325 & 0.416 \\
\hline & & & & & & &
\end{tabular}




\subsection{Results of the scaled regularized MVE model}

The optimal weights of the 20 stocks are obtained by solving model $S_{1}$ with their corresponding EVaR and variance values. Given the characteristics of the riskless asset, we restrict the 4-Week US Treasury Bill through this constraint: $0 \leq y \leq 0.2$. This restriction is imposed because of the riskless nature of the US Treasury Bill. Stocks and Treasury Bills are two different asset classes, the model will prefer allocating most of the amount invested to Treasury Bills because of its riskfree factor. It is appropriate to constrain the Treasury Bills. The Treasury Bills constraint is reflective of $\tilde{y}$ in constraint (18). The results are displayed in Table 3.

Table 3. Optimal weights $\left(x_{k}, y\right), k=1,2, \ldots, 20$ with corresponding EVaR and variance values among other outputs of the portfolios under varied required returns

\begin{tabular}{|c|c|c|c|c|c|c|c|c|c|}
\hline$R$ & 0.007000 & 0.007500 & 0.007800 & 0.008200 & 0.008800 & 0.009800 & 0.010400 & 0.012500 & 0.013000 \\
\hline$x_{1}$ & 0.000000 & 0.000000 & 0.000000 & 0.020227 & 0.054454 & 0.110908 & 0.144090 & 0.227485 & 0.232383 \\
\hline$x_{2}$ & 0.000000 & 0.000000 & 0.000000 & 0.000000 & 0.000000 & 0.000000 & 0.000000 & 0.000000 & 0.000000 \\
\hline$x_{3}$ & 0.008143 & 0.025952 & 0.031475 & 0.018315 & 0.007124 & 0.000000 & 0.000000 & 0.003276 & 0.006802 \\
\hline$x_{4}$ & 0.151133 & 0.150918 & 0.151362 & 0.152331 & 0.151248 & 0.142105 & 0.132057 & 0.078038 & 0.064679 \\
\hline$x_{5}$ & 0.000000 & 0.000000 & 0.000000 & 0.000000 & 0.000000 & 0.000000 & 0.000000 & 0.000000 & 0.000000 \\
\hline$x_{6}$ & 0.046299 & 0.059460 & 0.063656 & 0.054745 & 0.046511 & 0.034912 & 0.029932 & 0.024837 & 0.023792 \\
\hline$x_{7}$ & 0.066640 & 0.077167 & 0.080919 & 0.078278 & 0.074333 & 0.067223 & 0.063074 & 0.050366 & 0.046509 \\
\hline$x_{8}$ & 0.084901 & 0.093102 & 0.095726 & 0.087109 & 0.079121 & 0.065077 & 0.056732 & 0.032453 & 0.027338 \\
\hline$x_{9}$ & 0.000000 & 0.000000 & 0.000000 & 0.000000 & 0.000000 & 0.000000 & 0.000000 & 0.000000 & 0.000000 \\
\hline$x_{10}$ & 0.000000 & 0.000000 & 0.000000 & 0.000000 & 0.000000 & 0.000000 & 0.000000 & 0.000000 & 0.000000 \\
\hline$x_{11}$ & 0.038283 & 0.052426 & 0.057102 & 0.050217 & 0.043321 & 0.034170 & 0.030781 & 0.030574 & 0.030139 \\
\hline$x_{12}$ & 0.000000 & 0.000000 & 0.000000 & 0.000000 & 0.000000 & 0.000000 & 0.000000 & 0.043486 & 0.077165 \\
\hline$x_{13}$ & 0.000000 & 0.000000 & 0.000000 & 0.000000 & 0.000000 & 0.000000 & 0.000000 & 0.000000 & 0.000000 \\
\hline$x_{14}$ & 0.136651 & 0.138436 & 0.139940 & 0.147337 & 0.150664 & 0.148778 & 0.142892 & 0.098686 & 0.085997 \\
\hline$x_{15}$ & 0.000000 & 0.000000 & 0.000000 & 0.000000 & 0.000000 & 0.000000 & 0.000000 & 0.000000 & 0.000000 \\
\hline$x_{16}$ & 0.000000 & 0.002508 & 0.010056 & 0.012068 & 0.011222 & 0.014613 & 0.020218 & 0.052049 & 0.056563 \\
\hline$x_{17}$ & 0.150215 & 0.150247 & 0.150998 & 0.155517 & 0.156904 & 0.151341 & 0.142996 & 0.090858 & 0.077103 \\
\hline$x_{18}$ & 0.000000 & 0.007012 & 0.014568 & 0.019398 & 0.020415 & 0.026047 & 0.032403 & 0.063131 & 0.066840 \\
\hline$x_{19}$ & 0.000000 & 0.000000 & 0.000000 & 0.000000 & 0.000000 & 0.000000 & 0.000000 & 0.000000 & 0.000000 \\
\hline$x_{20}$ & 0.000000 & 0.000000 & 0.000000 & 0.000000 & 0.000000 & 0.000000 & 0.000000 & 0.000000 & 0.000000 \\
\hline$y$ & 0.200000 & 0.200000 & 0.200000 & 0.200000 & 0.200000 & 0.200000 & 0.200000 & 0.200000 & 0.200000 \\
\hline$x^{T} Q x$ & 0.000497 & 0.000596 & 0.000652 & 0.000667 & 0.000699 & 0.000787 & 0.000863 & 0.001300 & 0.001400 \\
\hline $\mathrm{EVaR}_{0.95}$ & 0.076600 & 0.086500 & 0.091900 & 0.094800 & 0.099400 & 0.108000 & 0.113700 & 0.137900 & 0.145300 \\
\hline $1-\left(e^{T} x+y\right)$ & 0.117700 & 0.042800 & 0.004200 & 0.004500 & 0.004700 & 0.004800 & 0.004800 & 0.004800 & 0.004700 \\
\hline$e^{T} x+y$ & 0.882300 & 0.957200 & 0.995800 & 0.995500 & 0.995300 & 0.995200 & 0.995200 & 0.995200 & 0.995300 \\
\hline
\end{tabular}




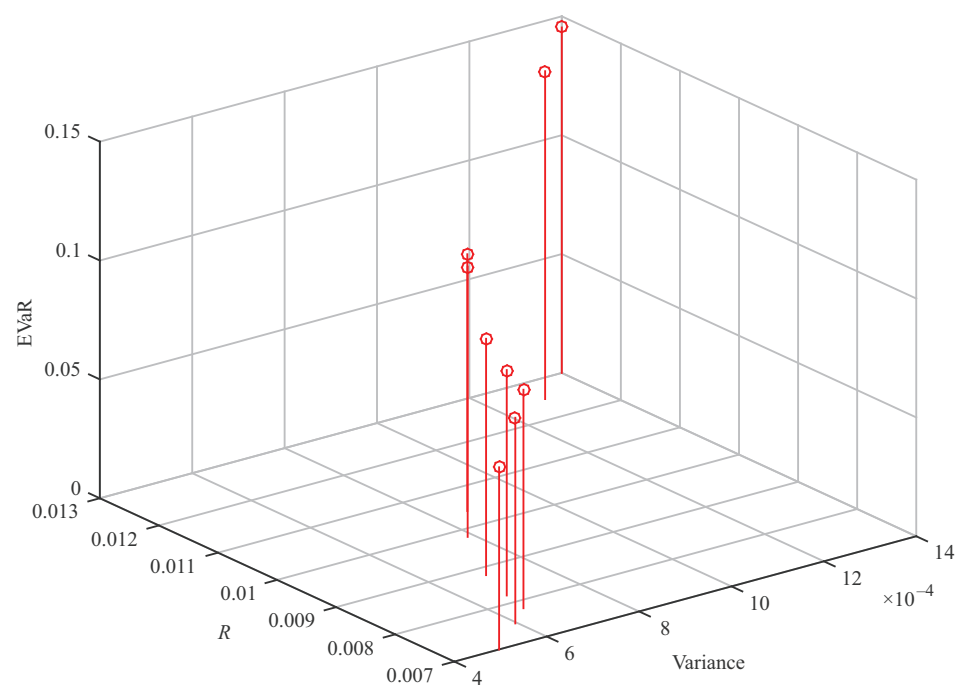

Fig. 1. The scaled regularized mean-variance-EVaR efficient frontier

From the results displayed by Table 3, the scaled regularized MVE frontier of the portfolio is graphically shown in Figure 1. A careful observation of Table 3 and Figure 1 show that as required returns $R$ increases, $\mathrm{EVaR}_{0.95}$ and variance values also increase, signifying that a higher return leads to higher risk, which is in line with existing literature and practice.

To illustrate the characteristic difference between the scaled regularized MVE model and the unscaled one, we compare output results such as risk measures, transaction costs $\left(1-\left(e^{T} x+y\right)\right)$ and capital amount invested $\left(e^{T} x+y\right)$ for some given level of $R$. We can clearly see from Table 3 and Table 4 that the transaction costs paid to implement the optimal solution to $S_{0}$ is lower than that of $P_{0}$. Therefore, the capital amount invested in portfolio $S_{0}$ is much higher than $P_{0}$ for given levels of $R$.

In Table 3, the variance is smaller than Table 4 but the EVaR is larger than in Table 3 when compared to Table 4. Thus, even though the variance is smaller, investors will have to be subjected to some additional risk in reducing the probability that the portfolio will incur large losses in order to pay lower transactions costs and have a chunk of their capital invested. In other words, investors would have to incur a small risk in controlling the downside risk of portfolio return if they want to pay lower transaction costs and have a large portion of their capital invested. Investors will be compensated with a smaller risk associated with the deviation around the expected return.

Table 4. Characteristic results of unscaled regularized MVE model

\begin{tabular}{lllll}
\hline \multicolumn{1}{c}{$R$} & 0.009800 & 0.010400 & 0.012500 & 0.013000 \\
\hline$x^{T} Q x$ & 0.000904 & 0.000983 & 0.001300 & 0.001400 \\
\hline $\mathrm{EVaR}_{0.95}$ & 0.084900 & 0.094500 & 0.131500 & 0.142000 \\
\hline $1-\left(e^{T} x+y\right)$ & 0.455400 & 0.409200 & 0.188900 & 0.112900 \\
\hline$e^{T} x+y$ & 0.544600 & 0.590800 & 0.811100 & 0.887100 \\
\hline
\end{tabular}




\subsection{Additional example}

To verify our findings, this study subjects our model to a larger dataset. This research uses the same dataset employed by Filomena and Lejeune (2012). The monthly stock price of 2570 stocks traded on NYSE, Amex and NASDAQ from January 29, 1999 to December 31, 2008 extracted via Wharton Research Data Service by Filomena and Lejeune (2012) was used. For the riskless asset, we consider the 3-Month US Treasury Bill rate on a monthly basis for the same period.

The initial position of stocks $x_{k}^{0}, k=1, \ldots, 2570$ is computed by using natural logarithm of the price ratio for 2 consecutive months from January 29, 1999 to December 31, 1999. Assuming the initial position for the 3-Month US Treasury Bill is zero i.e. $y^{0}=$ 0 , the initial position of stocks $x_{k}^{0}, k=1, \ldots, 2570$ is obtained by solving the standard mean-variance problem $B_{0}$.

We construct the portfolio invested in the 2570 stocks after revision based on natural logarithm of the price ratio for 2 consecutive days from January 31, 2000 to December 31, 2008 given that the amount invested in 3-Month US Treasury Bill after revision is constrained by $0 \leq y \leq 0.2$. Considering varied required returns, $R=0.0098,0.0104,0.0125,0.0130$, for $\epsilon=0.05, \psi=0.03$ and transaction costs, $c^{b}=c^{s}=0.02$, the optimal weights of the 2570 stocks are obtained by solving model $S_{1}$.

Table 5. Results of scaled regularized MVE model for 2570 stocks

\begin{tabular}{lllll}
\hline \multicolumn{1}{c}{$R$} & 0.009800 & 0.010400 & 0.012500 & 0.013000 \\
\hline \multicolumn{5}{c}{ Scaled regularized MVE model } \\
\hline$x^{T} Q x$ & 0.000179 & 0.000184 & 0.000231 & 0.000249 \\
\hline $\mathrm{EVaR}_{0.95}$ & 0.006610 & 0.007050 & 0.008660 & 0.090500 \\
\hline $1-\left(e^{T} x+y\right)$ & 0.003300 & 0.005100 & 0.003600 & 0.003600 \\
\hline$e^{T} x+y$ & 0.996700 & 0.994900 & 0.996400 & 0.996400 \\
\hline$x^{T} Q x$ & Unscaled regularized MVE model \\
\hline $\mathrm{EVaR}_{0.95}$ & 0.000208 & 0.000256 & 0.000231 & 0.000249 \\
\hline $1-\left(e^{T} x+y\right)$ & 0.627200 & 0.599100 & 0.499100 & 0.475300 \\
\hline$e^{T} x+y$ & 0.372800 & 0.400900 & 0.500900 & 0.524700 \\
\hline
\end{tabular}

From Table 5, we observe that as return increases, EVaR and variance values also increase. This signifies that a higher return leads to higher risk and it's in line with our earlier finding. The variance is smaller for the scaled regularized MVE model than the unscaled regularized MVE model, but the EVaR is larger than in the scaled regularized MVE model when compared to the unscaled one. This observation is in line with our earlier observation and confirms that investors would have to incur additional risk in controlling the downside risk of portfolio return if they want to pay lower transaction costs and have a significant portion of their capital invested. However, they will be compensated with having a lower risk associated with the deviation around the expected return. 


\section{Conclusions}

In this paper, we presented a scaled regularized mean-variance-EVaR with proportional transaction costs portfolio selection model, which selects a solution based on three parameters, which are variance, EVaR and the expected return. This model is one of a multi-objective type: expected return is maximized, and variance and EVaR are minimized. We chose variance and EVaR because variance measures the deviation from the expected return whiles EVaR will help control the downside risk of portfolio return.

In pursuing the solution of this multi-objective model, we reduce it to a minimization problem in which variance and EVaR are minimized whiles constraints are imposed on the expected return. Random returns are usually represented by the mean estimate, which leads to estimation risk. We imposed the squared $l_{2}$-norm ball constraint to deal with this problem.

For a varied level of required returns, we obtain efficient solutions of the scaled regularized mean-variance-EVaR model. In a variance-EVaR space, our scaled regularized standard risk minimization formulated model obtains a range of efficient solutions which forms a curve that represents an optimal compromise between short tails and the risk associated with the deviation around expected return. Our model lowers transaction costs and increases the amount of capital to be used for investment. Even though the variance is smaller, investors will have to incur some additional risk in reducing the probability that the portfolio will incur large losses if they want to pay lower transactions costs and have a chunk of their capital invested.

The scaled regularized mean-variance-EVaR model represents a compromise between traditional fund managers' need for small variance and regulators' necessity for short tails. Our model can also help the investor have a chunk of his capital invested. For a given required return, one can obtain the optimal allocation of assets in the portfolio and enhance the efficiency of portfolio selection by controlling the overall risk.

The methodology presented in this research is based on some assumptions on asset characteristics such as the kind of assets and its distributional properties. One possible future research approach would be to consider, instead of Gaussian distributed returns of stocks, the more general alpha-stable distributed returns of stocks, which could lead to a better understanding from a financial perspective. Our approach may be extended for a portfolio that is made of several or different assets under different financial market situations with different realistic assumptions. It is also possible to use the results from other methodologies to compare results of our model under the scope of computing times and efficiency.

\section{Acknowledgements}

We thank the reviewers for taking time to add their comments and suggestions to this paper. We acknowledge support by the National Nature Science Foundation of China (11571061). We thank Glory George-Ufot for her valuable inputs. Special thanks goes out to Journal of Business Economics and Management for providing us the platform to contribute to literature. 


\section{Funding}

This work was supported by the National Nature Science Foundation of China (11571061).

\section{Disclosure statement}

The authors declare that they have no competing interests.

\section{References}

Ahmadi-Javid, A. 2012. Entropic value-at-risk: a new coherent risk measure, Journal of Optimization Theory and Applications 155(3): 1105-1123. https://doi.org/10.1007/s10957-011-9968-2

Ahmed, S. 2006. Convexity and decomposition of mean-risk stochastic programs, Mathematical Programming 106(3): 433-446. https://doi.org/10.1007/s10107-005-0638-8

Agarwal, V.; Naik, N. Y. 2004. Risks and portfolio decisions involving hedge funds, Review of Financial Studies 17(1): 63-98. https://doi.org/10.1093/rfs/hhg044

Artzner, P.; Delbaen, F.; Eber, J. M; Heath, D. 1999. Coherent measures of risk, Mathematical Finance 9(3): 203-228. https://doi.org/10.1111/1467-9965.00068

Atta Mills, E. F. E.; Yan, D.; Yu, B.; Wei, X. 2016a. Research on regularized meanvariance portfolio selection strategy with modified Roy safety-first principle, SpringerPlus 5(1): 919. https://doi.org/10.1186/s40064-016-2621-7

Atta Mills, E. F. E.; Yu, B.; Gu, L. 2016b. On meeting capital requirements with a chance constrained optimization model, SpringerPlus 5(1): 500. https://doi.org/10.1186/s40064-016-2110-z Axelrod, A.; Carlone, L.; Chowdhary, G.; Karaman, S. 2016. Data-driven prediction of EVAR with confidence in time-varying datasets, in Decision and Control (CDC), 2016 IEEE 55th Conference, 12-14 December 2016, Las Vegas, NV, USA, 5833-5838.

Bawa, V. S.; Brown, S. J.; Klein, R. W. 1979. Estimation risk and optimal portfolio choice. North-Holland Publishing Company, 190.

Best, M. J.; Grauer, R. R. 1991. Sensitivity analysis for mean-variance portfolio problems, Management Science 37(8): 980-989. https://doi.org/10.1287/mnsc.37.8.980

Black, F.; Litterman, R. 1992. Global portfolio optimization, Financial Analysts Journal 48(5): 28-43. https://doi.org/10.2469/faj.v48.n5.28

Borkovec, M.; Domowitz, I.; Kiernan, B.; Serbin, V. 2010. Portfolio optimization and the cost of trading, The Journal of Investing 19(2): 63-76. https://doi.org/10.3905/joi.2010.19.2.063

Brodie, J.; Daubechies, I.; De Mol, C.; Giannone, D.; Loris, I. 2009. Sparse and stable Markowitz portfolios, Proceedings of the National Academy of Sciences 106(30): 12267-12272. https://doi.org/10.1073/pnas.0904287106

Caccioli, F.; Kondor, I.; Marsili, M.; Still, S. 2016. Liquidity risk and instabilities in portfolio optimization, International Journal of Theoretical and Applied Finance 19(05): 1650035. https://doi.org/10.1142/S0219024916500357

Ceria, S.; Stubbs, R. A. 2006. Incorporating estimation errors into portfolio selection: robust portfolio construction, Journal of Asset Management 7(2): 109-127.

https://doi.org/10.1057/palgrave.jam.2240207

Charnes, A.; Cooper, W. W. 1962. Programming with linear fractional functionals, Naval Research Logistics Quarterly 9(3-4): 181-186. https://doi.org/10.1002/nav.3800090303 
Chen, A. H.; Jen, F. C.; Zionts, S. 1971. The optimal portfolio revision policy, The Journal of Business 44(1): 51-61. https://doi.org/10.1086/295332

Cherny, A. S.; Madan, D. B. 2006. Coherent measurement of factor risks, arXiv preprint math/0605062.

Chiam, S. C.; Tan, K. C.; Mamun, A. A. 2009. A memetic model of evolutionary PSO for computational finance applications, Expert Systems with Applications 36(2): 3695-3711.

Chopra, V. K.; Ziemba, W. T. 2011. The effect of errors in means, variances, and covariances on optimal portfolio choice, in L. C. MacLean, E. O. Thorp, W. T. Ziemba (Eds). The Kelly Capital growth investment criterion: theory and practice. Volume 3. World Scientific Publishing Co. Pte. Ltd.

Chunhachinda, P.; Dandapani, K.; Hamid, S.; Prakash, A. J. 1997. Portfolio selection and skewness: evidence from international stock markets, Journal of Banking \& Finance 21(2): 143-167. https://doi.org/10.1016/S0378-4266(96)00032-5

Dai, Z.; Wen, F. 2014. Robust CVaR-based portfolio optimization under a general affine data perturbation uncertainty set, Journal of Computational Analysis \& Applications 16(1): 93-103.

DeMiguel, V.; Garlappi, L.; Nogales, F. J; Uppal, R. 2009. A generalized approach to portfolio optimization: improving performance by constraining portfolio norms, Management Science 55(5): 798-812. https://doi.org/10.1287/mnsc.1080.0986

Dumas, B.; Luciano, E. 1991. An exact solution to a dynamic portfolio choice problem under transactions cost, The Journal of Finance 46(2): 577-595.

https://doi.org/10.1111/j.1540-6261.1991.tb02675.x

Dybvig, P. H. 2005. Mean-variance portfolio rebalancing with transaction costs. Working paper, Washington University in Saint Louis.

Economic Research - St.Louis Fed. 2016. FRED Economic data [online], [cited 06 September 2016]. Economic Research of the Federal Reserve Bank of St. Louis. Available from Internet: https://fred.stlouisfed.org/series/TB4WK

Fan, J.; Zhang, J.; Yu, K. 2012. Vast portfolio selection with gross-exposure constraints, Journal of the American Statistical Association 107(498): 592-606.

https://doi.org/10.1080/01621459.2012.682825

Föllmer, H.; Schied, A. 2004. Stochastic finance. de Gruyter Studies in Mathematics 27.

Filomena, T. P; Lejeune, M. A. 2012. Stochastic portfolio optimization with proportional transaction costs: convex reformulations and computational experiments, Operations Research Letters 40(3): 212-217. https://doi.org/10.1016/j.orl.2012.01.003

Gaivoronski, A. A.; Pflug, G. 2005. Value-at-risk in portfolio optimization: properties and computational approach, Journal of Risk 7(2): 1-31. https://doi.org/10.21314/JOR.2005.106

Goldfarb, D.; Iyengar, G. 2003. Robust portfolio selection problems, Mathematics of Operations Research 28(1): 1-38. https://doi.org/10.1287/moor.28.1.1.14260

Ghosh, A.; Mahanti, A. 2014. Investment portfolio management: a review from 2009 to 2014, in Proceedings of the 10th Global Business and Social Science Research Conference, 23-24 May 2014, Beijing, China.

Green, R. C.; Hollifield, B. 1992. When will mean-variance efficient portfolios be well diversified?, The Journal of Finance 47(5): 1785-1809.

https://doi.org/10.1111/j.1540-6261.1992.tb04683.x

Hester, D. D.; Tobin, J. 1967. Studies of portfolio behavior. John Wiley \& Sons 20.

Huang, X. 2008. Mean-semivariance models for fuzzy portfolio selection, Journal of Computational and Applied Mathematics 217(1): 1-8. https://doi.org/10.1016/j.cam.2007.06.009 
Jagannathan, R.; Ma, T. 2003. Risk reduction in large portfolios: why imposing the wrong constraints helps, The Journal of Finance 58(4): 1651-1684. https://doi.org/10.1111/1540-6261.00580 Jobson, J. D.; Korbie, B. 1980. Estimation for Markowitz efficient portfolios, Journal of the American Statistical Association 75(371): 544-554.

https://doi.org/10.1080/01621459.1980.10477507

Jorion, P. 1986. Bayes-Stein estimation for portfolio analysis, Journal of Financial and Quantitative Analysis 21(03): 279-292. https://doi.org/10.2307/2331042

Kao, C.; Steuer, R. E. 2016. Value of information in portfolio selection, with a Taiwan stock market application illustration, European Journal of Operational Research 253(2): 418-427. https://doi.org/10.1016/j.ejor.2016.02.011

Kellerer, H.; Mansini, R.; Speranza, M. G. 2000. Selecting portfolios with fixed costs and minimum transaction lots, Annals of Operations Research 99(1-4): 287-304.

https://doi.org/10.1023/A:1019279918596

Kendall, G.; Su, Y. 2005. A particle Swarm optimisation approach in the construction of optimal risky portfolios, in Proceedings of the 23rd IASTED International Multi-Conference Artificial Intelligence and Applications, 14-16 February 2005, Innsbruck, Austria, 2005. Artificial Intelligence and Applications 453: 140-145.

Konno, H.; Shirakawa, H.; Yamazaki, H. 1993. A mean-absolute deviation-skewness portfolio optimization model, Annals of Operations Research 45(1): 205-220.

https://doi.org/10.1007/BF02282050

Konno, H.; Suzuki, K. 1995. A mean-variance-skewness portfolio optimization model, Journal of the Operations Research Society of Japan 38(2): 173-187.

Krokhmal, P.; Palmquist, J.; Uryasev, S. 2002. Portfolio optimization with conditional value atrisk objective and constraints, Journal of Risk 4: 43-68. https://doi.org/10.21314/JOR.2002.057

Ledoit, O.; Wolf, M. 2004. A well-conditioned estimator for large-dimensional covariance matrices, Journal of Multivariate Analysis 88(2): 365-411.

https://doi.org/10.1016/S0047-259X(03)00096-4

León, T.; Liern, V.; Vercher, E. 2002. Viability of infeasible portfolio selection problems: a fuzzy approach, European Journal of Operational Research 139(1): 178-189.

https://doi.org/10.1016/S0377-2217(01)00175-8

Lintner, J. 1965. Security prices, risk, and maximal gains from diversification, The Journal of Finance 20(4): 587-15. https://doi.org/10.1111/j.1540-6261.1965.tb02930.x

Lobo, M. S.; Fazel, M.; Boyd, S. 2007. Portfolio optimization with linear and fixed transaction costs, Annals of Operations Research 152(1): 341-365.

Loraschi, A.; Tomassini, M.; Tettamanzi, A.; et al. 1995. Distributed genetic algorithms with an application to portfolio selection problems, in Proceedings of the 23rd IASTED International Multi-Conference Artificial Intelligence and Applications, 14-16 February 2005. Artificial Neural Nets And Genetic Algorithms, 384-387.

Mansini, R.; Ogryczak, W.; Speranza, M. G. 2014. Twenty years of linear programming based portfolio optimization, European Journal of Operational Research 234(2): 518-535.

https://doi.org/10.1016/j.ejor.2013.08.035

Maringer, D. G. 2006. Portfolio management with heuristic optimization. Springer Science \& Business Media 8.

Markowitz, H. 1952. Portfolio selection, The Journal of Finance 7(1): 77-91.

https://doi.org/10.1111/j.1540-6261.1952.tb01525.x

Markowitz, H. M. 1968. Portfolio selection: efficient diversification of investments. Yale University Press. 
Markowitz, H.; Todd, P.; Xu, G.; Yamane, Y. 1993. Computation of mean-semi variance efficient sets by the critical line algorithm, Annals of Operations Research 45(1): 307-317.

https://doi.org/10.1007/BF02282055

Merton, R. C. 1980. On estimating the expected return on the market: an exploratory investigation, Journal of Financial Economics 8(4): 323-361. https://doi.org/10.1016/0304-405X(80)90007-0

Mitchell, J. E.; Braun, S. 2004. Rebalancing an investment portfolio in the presence of convex transaction costs (unpublished source).

Mitchell, J. E.; Braun, S. 2013. Rebalancing an investment portfolio in the presence of convex transaction costs, including market impact costs, Optimization Methods and Software 28(3): 523-542. https://doi.org/10.1080/10556788.2012.717940

Muthuraman, K.; Kumar, S. 2006. Multidimensional portfolio optimization with proportional transaction costs, Mathematical Finance 16(2): 301-335.

https://doi.org/10.1111/j.1467-9965.2006.00273.x

Moazeni, S.; Coleman, T. F.; Li, Y. 2016. Smoothing and parametric rules for stochastic mean CVaR optimal execution strategy, Annals of Operations Research 237(1-2): 99-120.

https://doi.org/10.1007/s10479-013-1391-7

Nagai, H. 2003. Optimal strategies for risk-sensitive portfolio optimization problems for general factor models, SIAM Journal on Control and Optimization 41(6): 1779-800.

https://doi.org/10.1137/S0363012901399337

Oksendal, B.; Sulem, A. 2002. Optimal consumption and portfolio with both fixed and proportional transaction costs, SIAM Journal on Control and Optimization 40(6): 1765-1790.

https://doi.org/10.1137/S0363012900376013

Philippatos, G. C.; Wilson, C. J. 1972. Entropy, market risk, and the selection of efficient portfolios, Applied Economics 4(3): 209-220. https://doi.org/10.1080/00036847200000017

Pogue, G. A. 1970. An extension of the Markowitz portfolio selection model to include variable transaction costs, shorts sales, leverage policies and taxes, Journal of Finance 25(5): 1005-1027. https://doi.org/10.1111/j.1540-6261.1970.tb00865.x

Price, K.; Price, B.; Nantell, T. J. 1982. Variance and lower partial moment measures of systematic risk: some analytical and empirical results, The Journal of Finance 37(3): 843-855.

https://doi.org/10.1111/j.1540-6261.1982.tb02227.x

Rockafellar, R. T.; Uryasev, S. 2000. Optimization of conditional value-at-risk, Journal of Risk 2: 21-42. https://doi.org/10.21314/JOR.2000.038

Rockafellar, R. T.; Uryasev, S. 2002. Conditional value-at-risk for general loss distributions, Journal of Banking \& Finance 26(7): 1443-1471. https://doi.org/10.1016/S0378-4266(02)00271-6

Roman, D.; Darby-Dowman, K.; Mitra, G. 2007. Mean-risk models using two risk measures: a multi-objective approach, Quantitative Finance 7(4): 443-458.

https://doi.org/10.1080/14697680701448456

Samuelson, P. A. 1969. Lifetime portfolio selection by dynamic stochastic programming, The Review of Economics and Statistics 1: 239-246. https://doi.org/10.2307/1926559

Schaible, S. 1974. Parameter-free convex equivalent and dual programs of fractional programming problems, Zeitschrift for Operations Research 18: 187-196. https://doi.org/10.1007/bf02026600

Schreiner, J. 1980. Portfolio revision: a turnover-constrained approach, Financial Management 1: 67-75. https://doi.org/10.2307/3665315

Shalit, H.; Yitzhaki, S. 1984. Mean-Gini, portfolio theory, and the pricing of risky assets, The Journal of Finance 39(5): 1449-1468. https://doi.org/10.1111/j.1540-6261.1984.tb04917.x

Sharpe, W. F. 1964. Capital asset prices: a theory of market equilibrium under conditions of risk, The Journal of Finance 19(3): 425-442. https://doi.org/10.1111/j.1540-6261.1964.tb02865.x 
Sharpe, W. F. 1967. A linear programming algorithm for mutual fund portfolio selection, Management Science 13(7): 499-510. https://doi.org/10.1287/mnsc.13.7.499

Tanaka, H.; Guo, P.; Türksen, I. B. 2000. Portfolio selection based on fuzzy probabilities and possibility distributions, Fuzzy Sets and Systems 111(3): 387-397.

https://doi.org/10.1016/S0165-0114(98)00041-4

Tütüncü, R. H.; Koenig, M. 2004. Robust asset allocation, Annals of Operations Research 132(14): 157-187. https://doi.org/10.1023/B:ANOR.0000045281.41041.ed

Vercher, E.; Bermúdez, J. D.; Segura, J. V. 2007. Fuzzy portfolio optimization under downside risk measures, Fuzzy Sets and Systems 158(7): 769-782. https://doi.org/10.1016/j.fss.2006.10.026

Wang, X. T.; Li, Z.; Zhuang, L. 2017. Risk preference, option pricing and portfolio hedging with proportional transaction costs, Chaos, Solitons \& Fractals 95: 111-130.

https://doi.org/10.1016/j.chaos.2016.12.010

Yao, H.; Li, Z.; Lai, Y. 2013. Mean-CVaR portfolio selection: a nonparametric estimation framework, Computers \& Operations Research 40(4): 1014-1022.

https://doi.org/10.1016/j.cor.2012.11.007

Zhao, S.; Lu, Q.; Han, L.; Liu, Y.; Hu, F. 2015. A mean-CVaR-skewness portfolio optimization model based on asymmetric Laplace distribution, Annals of Operations Research 226(1): 727-739. https://doi.org/10.1007/s10479-014-1654-y

Zheng, C.; Chen, Y. 2014. Portfolio selection based on relative entropy coherent risk measure, Systems Engineering - Theory \& Practice 34(3): 648-655.

Zhu, J. 2017. Optimal financing and dividend distribution with transaction costs in the case of restricted dividend rates, ASTIN Bulletin: The Journal of the IAA 47(1): 239-268.

Ebenezer Fiifi Emire ATTA MILLS is a Researcher and holds a PhD in Financial Mathematics and Actuarial Science from Dalian University of Technology. He holds a Masters degree in Finance from Jiangsu University. His research interests are portfolio optimization, risk modeling and econometrics.

Bo YU is a Professor in the School of Mathematical Sciences at Dalian University of Technology. His research interests include applied mathematics, numerical mathematics, computational mathematics and optimization.

Jie YU is a Master student in the School of Mathematical Sciences at Dalian University of Technology. Her research interest includes portfolio optimization and risk management. 